\title{
Incidence of breast and nipple abnormalities among primigravid women in Sri Lanka
}

\author{
Sujeewa Amarasena ${ }^{1}$ \\ Sri Lanka Journal of Child Health, 2006; 35: 51-4
}

(Key words: incidence, breast and nipple abnormalities, primigravid women, Sri Lanka)

\begin{abstract}
Objective To determine the incidence of breast and nipple abnormalities among primigravid women and their effects on lactation in Sri Lanka.

Design Prospective descriptive survey.

Method All primigravid women attending antenatal clinics of the university obstetric unit, giving informed consent, were recruited into the study. Their breasts were examined clinically and breast abnormalities noted. They were followed up until successful lactation was established.

Results 956 mothers were examined. 768 had normal breasts and 188 abnormalities were noted. 725 completed the study. $72.5 \%$ established successful lactation. 80 had flat nipples of whom 52 completed the study. 44 flat nipples were corrected antenatally with exercises. All established lactation successfully. Other abnormalities included breast surgery, hypoplastic breasts or breast asymmetry. 9.8\% of women with breast/nipple abnormalities failed to establish lactation.
\end{abstract}

Conclusion Large majority (84.6\%) of flat non protractile nipples are correctable with antenatal exercises.

\section{Introduction}

Breast and nipple abnormalities are commonly encountered in clinical practice especially in relation to lactation problems postnatally ${ }^{1}$. These abnormalities include flat or inverted nipples, fissured nipples, breast sugery ${ }^{2}$, breast radiation ${ }^{3}$, marked breast asymmetry (micromastia) hypoplastic

${ }^{1}$ Senior lecturer and Head, Department of Paediatrics, Faculty of Medicine, Galle

(Received on 8 August 2005. Accepted on 10 September 2005) breast $^{4}$, unilateral or bilateral absence of breast (amastia) and tubular breast deformity ${ }^{1}$. These are well-recognized causes of lactation failure. Some abnormalities are correctable with antenatal interventions. One study identified the incidence of inverted or nonprotractile nipples resulting in attachment difficulties to be $10 \%$ among 1926 nulliparous women ${ }^{5}$. Incidence of these abnormalities and their correctability among Sri Lankan women is not known.

\section{Objectives}

1. To assess the incidence of breast and nipple abnormalities among primigravid women attending antenatal clinics in Sri Lanka.

2. To assess the correctability of flat or inverted nipples with Hoffman ${ }^{4}$ exercises.

3. To assess the effects of antenatal interventions on corrected and persistent abnormalities on successful lactation.

\section{Setting}

Study was conducted at antenatal clinics (ANCs) and postnatal wards of the university obstetric unit, Teaching Hospital Mahamodara and special care baby unit (SCBU) and well baby clinic of the university paediatric unit, Teaching Hospital Mahamodara and Teaching Hospital Karapitiya from 20.03.2000 to 24.02.2001. Ethics committee of the Faculty of Medicine, Galle, granted ethical clearance.

\section{Method}

All primigravid women attending ANCs were given a leaflet explaining the objectives of the study. Those giving written informed consent were enrolled into the study. The author examined the breasts. Research assistants filled data into questionnaires. The women were categorized as having normal or abnormal 
breasts. Those with inverted or flat non-protractile nipples were prescribed nipple exercises as described by Hoffmann ${ }^{5}$. Those with normal breasts were allowed routine follow-up at the ANC and interventions were to support or provide breastfeeding counselling as part of the routine care postnatally. Both groups were provided with education on the need for exclusive breast-feeding (EBF) for four months. Infants were followed up in the well baby clinic for 4 months of postnatal age which was the end point of the study. Those women advised on nipple exercises were seen at the next routine clinic visit for clinical evidence of correction of the abnormality. Compliance with exercise regimen was checked. They were also followed up until delivery \& provided with breast-feeding counselling/support postnatally. Registered letters were posted to given addresses of women who were lost to follow up. Attempts were made to trace these women through the midwives of the relevant area. Babies were followed up for 4 months for EBF \& satisfactory weight gain. Successful lactation was defined as EBF for 4 months with satisfactory weight gain. Satisfactory weight gain was defined as having a trend to double the birth weight by 4-5 months or following weight curve on child health development record (CHDR) or 150-200g weight gain per week on calculation. Incidence of lactation failure and reasons were recorded. Hypoplastic breasts were defined as small breasts, small areolae and small nipples with minimal breast changes in pregnancy followed by less/minimal milk secretion on the affected $\operatorname{side}^{2}$. During follow up other confounding factors interfering with lactation were identified and support was provided to overcome these. Epi info 6 was used for statistical analysis.

\section{Results}

A total of 956 primigravid women were examined. Out of 956 women 788 had normal breasts $(80.3 \%)$. $188(19.7 \%)$ had breast abnormalities. A total of 725 (75.8\%) reached the end point of the study including the thirteen who were traced with letters. Of these $593(77.2 \%)$ were from the normal group and 132 $(70.2 \%)$ were from the abnormal group.

Breast abnormalities included surgery 10/956 (1\%), flat non-protractile nipples 80/956 (8.3\%), inverted nipple $12 / 956(1.3 \%) \quad \&$ asymmetric/hypoplastic breasts $86 / 956(8.9 \%)$.

In the breast surgery group 3/10 had sub areolar incisions and 7 were outside the areola. It was unilateral in 9 and bilateral in one. 9/10 completed the study. 6 established lactation successfully. Three failed despite surgery being unilateral and all 3 were sub areolar incisions.

$52 / 80 \quad(75 \%)$ with flat non-retractile nipples completed the study. 43 were left-sided and 11 were right-sided. 26 were bilateral. 44 were corrected antenatally with nipple exercises. It was corrected within 2 weeks in 14, within 2-4 weeks in 25 and within 4-6 weeks in 5. Eight failed correction. Compliance on exercise regimen was satisfactory.

All 44 women with corrected flat nipples established lactation successfully. Only $3 / 8$ of the uncorrected group established lactation successfully. Only 3/12 women with inverted nipples completed the study. It was corrected in 2 in 8 weeks but failed in one. But all 3 failed to established lactation successfully. $61 / 86$ of the asymmetry/hypoplastic group completed the study. Asymmetry/hypoplasia was right sided in 38 and left sided in 48. In the follow up group 34 were left sided and 27 were right sided. On follow up $29 / 61$ were categorized into asymmetry group and $32 / 61$ into hypoplastic group. 29/29 of the asymmetry group and $30 / 32$ of the hypoplastic group established lactation successfully. 2/32 (6\%) with hypoplasia failed to establish lactation. Incidence of breast hypoplasia was $3.3 \%$ (32/956) among the original recruits. Incidence of lactation failure among women with abnormalities was $9.8 \%$ and in the normal group $4.4 \%(32 / 725)$. This difference is not statistically significant $(\mathrm{P}>0.05)$. As a group $72.5 \%$ of original 956 established successful lactation. 29 of 188 mothers with abnormalities were assigned to asymmetry group on follow up and if this is excluded incidence of nipple/breast abnormalities would be $16.3 \%$.

\section{Discussion}

Nipple \& breast abnormalities are recognized causes of feeding problems leading to lactation failure. Hospitals and maternity units where deliveries take place need to provide a support service to ensure successful establishment of breast-feeding prior to discharge and continued breast-feeding support system after discharge. This study attempted to identify nipple or breast abnormalities with a potential to cause feeding problems leading to lactation failure antenatally and provide interventions to correct abnormalities.

Eighty cases of flat/non-protractile nipples were detected of whom 52 completed the study. All were prescribed Hoffmann exercises but only 44 were corrected. All of them established lactation successfully. In the 8 which failed correction, 5 failed 
to establish lactation. Although this is a small number to compare, the difference is statistically significant. This was despite good compliance with nipple exercises. All were due to poor attachment and difficulties in feeding leading to loss of confidence, impaired milk transfer and insufficient milk production. Difficulties in getting attachment with extreme flat nipples is demonstrated by the failure in $5 / 8$ mothers with persistent abnormalities. Even with correction it may be difficult as reflected by all 3 failures in the inverted group.

Even with support, counselling and syringe technique prescribed to manage flat non-protractile nipples good attachment was difficult to obtain. This, with our mother's desire to go home early from the crowded hospital setup, pressurizes the staff to discharge early which may result in increased likelihood to bottle feed at home after sometime with feeding problems. This is especially so with the time taken for correction of the abnormality. If mothers have to work hard together with health workers for 26 weeks to get a good attachment, the entire process is likely to fail. This is due to stress, pain and discomfort, lack of family and trained health care workers support leading to loss of motivation and confidence in breast feeding in the mother. Two common abnormalities namely flat nipples \& breast asymmetry were found to be more common on left side. This cannot be explained. Even with breast asymmetry successful breast-feeding can be established in a majority and minimal asymmetry is considered a normal variation. However marked asymmetry, if present, may indicate glandular hypoplasia. Even with unilateral hypoplastic breasts lactation could be established successfully. However, some will fail like $2 / 32(4.4 \%)$ in this study. Diaphonography ${ }^{4}$ has been used to diagnose glandular hypoplasia. The test is to transilluminate the breasts and a difference in transillumination is seen with milkless breast being transilluminant and the breast full of milk being translucent. It is easy to manage this with supplements with an early diagnosis. Clinically hypoplastic breasts could be diagnosed using criteria such as smaller sized areola, nipple and minimal breast changes in pregnancy ${ }^{4}$. Mothers should be encouraged to breast feed and often are capable of producing an adequate milk supply to maintain satisfactory weight gain in a large majority if it is unilateral. Support should be provided to overcome emotions of not breast feeding their infants to those who fail.

The problem of having to counsel mothers with abnormal nipples/breasts for the first time during the immediate postnatal period was avoided in this study.
Hoffmann exercises are not regularly used in our antenatal clinics in the correction of flat non-protractile nipples or inverted nipples. Controversy surrounds the value of these exercises in the correction of the abnormalities and the negative effects of informing expectant women about abnormalities with nipples ${ }^{5}$. However, this study was designed to identify the value of the exercises as a result of this controversy. (52/80) $75 \%$ of mothers with flat nipples completed the study. Abnormality was corrected with 44/52 (84.6\%). All of them established lactation successfully. In contrast, out of $8 / 52$ in whom the abnormality was not corrected, 5 failed to establish lactation.

\section{Conclusions}

Breast and nipple abnormalities are common with an incidence of $16.3 \%$ among nullliparous women attending ANCs. Large majority $(90.2 \%)$ of them exclusively breast fed with satisfactory weight gain with adequate support. Incidence of flat/non protractile inverted nipples is $9.6 \%$ and marked asymmetry/hypoplasia is $5.7 \%$; breast surgery was found among $1 \%$ of women. Large majority of flat/ non protractile nipple can be corrected antenatally (75\%) with Hoffman exercises and they could successfully breast feed the offspring.

\section{Acknowledgments}

I thank Dr. M. Madarasinghe and demonstrators for helping to examine mothers and filling questionnaire and Dr. Gaya Wijerathna and Dr. Lahiru Prabodha for secretarial assistance.

\section{References}

1. Neifort M R. Clinical aspects of lactation. Clinics in perinatology 1999; 26: 283 - 304:

2. Neifort $M$, De Marzo S, Seacat $J$ et al. The influence of breast surgery, breast appearance and pregnancy induced breast changes on lactation sufficiency as measured by infant weight gain. Birth 1990; 17; 31.

3. Neifort M R. The optimization of breast feeding in the perinatal period. Clinics in perinatology 1998; 25: 303-20.

4. Neifort M, Lawrance R, Seacat J. Lactation failure due to insufficient glandular development of the breasts. Pediatrics $1985 ; 76: 823$

5. Alexander J, Grant A, Campbell M. Randomised control trial of breast shells and Hoffman exercises for inverted and non protractile nipples. BMJ 1992: 304: 1030 . 